\title{
Malaria prevalence, knowledge, perception, preventive and treatment behavior among military in Champasak and Attapeu provinces, Lao PDR: a mixed methods study
}

Phoutnalong Vilay ${ }^{1,2,3^{*}}$ D, Daisuke Nonaka ${ }^{1,3}$, Phosadeth Senamonty ${ }^{4}$, Malayvanh Lao ${ }^{4}$, Moritoshi Iwagami ${ }^{3,5,6}$, Jun Kobayashi ${ }^{1,3}$, Paul Michael Hernandez ${ }^{1,7}$, Ketkesone Phrasisombath ${ }^{8}$, Sengchanh Kounnavong ${ }^{3,9}$,

Bouasy Hongvanthong ${ }^{2,3}$, Paul T. Brey ${ }^{3,6}$ and Shigeyuki Kano ${ }^{3,5}$

\begin{abstract}
Background: Malaria is a major health problem in Lao People's Democratic Republic (Lao PDR) with high transmission in remote and forest areas, particularly in the South. The military is at risk of malaria infection especially those deployed in forest areas. This study determined the prevalence of malaria infection and assessed knowledge, perception, and preventive and treatment behavior regarding malaria among military personnel in two southern provinces in Lao PDR.

Methods: Quantitative and qualitative approaches were undertaken in Champasak and Attapeu provinces in 2017. From 313 military personnel, quantitative data were collected through questionnaire-based interviews and blood samples used for parasite detection by polymerase chain reaction (PCR). Qualitative data were collected through 7 focus group discussions and 17 in-depth interviews among 49 military personnel. Fisher's exact test and Mann-Whitney $U$ test were used to assess the association between malaria infection and participant characteristics. Content analysis for qualitative data was performed to explore perception and treatment behaviors regarding malaria.

Results: The prevalence of malaria infection was 11.2\% (Plasmodium falciparum: 1.3\%, Plasmodium vivax: 9.3\% and mixed infections: $0.6 \%$ ). Many participants understood that malaria is transmitted through mosquito bites, although they did not necessarily know the name of vector mosquitoes (Anopheles). Surprisingly, more than a half also believed that malaria is transmitted through drinking stream water. One-third of the participants used long-lasting insecticidal nets. Due to limited supply, participants were often unable to use mosquito repellent and coils when necessary. Because participants were unable to receive timely diagnosis and appropriate treatment for malaria in their camps, they commonly practiced self-treatment using antibiotics, painkillers, and/or traditional medicines. They only go to a healthcare facility through their supervisor if their conditions worsen.

Conclusions: The prevalence of symptomatic and asymptomatic malaria was conspicuous among military in forest areas. Many participants believed that malaria is transmitted not only by mosquito bites but also from drinking stream water. Preventive equipment was often insufficient. Self-treatment was practiced before referring to healthcare facility. To further prevent military from contracting malaria, the National Malaria Control Program and military body should provide adequate and suitable health education, protective equipment, and on-site malaria case management.
\end{abstract}

Keywords: Malaria, Military, Prevalence, Knowledge, Perception, Preventive measure, Treatment behavior, Laos

\footnotetext{
* Correspondence: phoutnalongvilay@gmail.com

1 Department of Global Health, Graduate School of Health Sciences,

University of the Ryukyus, 207 Uehara-cho, Okinawa 903-0215, Japan

${ }^{2}$ Center of Malariology, Parasitology and Entomology, Ministry of Health,

Vientiane, Lao PDR

Full list of author information is available at the end of the article
}

(c) The Author(s). 2019 Open Access This article is distributed under the terms of the Creative Commons Attribution 4.0 International License (http://creativecommons.org/licenses/by/4.0/), which permits unrestricted use, distribution, and reproduction in any medium, provided you give appropriate credit to the original author(s) and the source, provide a link to the Creative Commons license, and indicate if changes were made. The Creative Commons Public Domain Dedication waiver (http://creativecommons.org/publicdomain/zero/1.0/) applies to the data made available in this article, unless otherwise stated. 


\section{Introduction}

In Lao People's Democratic Republic (Lao PDR), malaria transmission is low in plains or high-altitude are as, and high in remote, hilly and forested areas particularly in the south [1]. Although the provision of long-lasting insecticide nets (LLINs) at highly subsidized price, and free screening and case management with artemisinin-based combination therapy have aided in reducing the incidence of malaria, malaria remains to be a serious public health problem in the southern part of Lao PDR [2]. Approximately $94 \%$ of malaria cases $(34,083 / 36,115)$ were reported from endemic areas in the south including Savannakhet, Saravanh, Sekong, Champasak, and Attapeu provinces in 2015. According to the malaria surveillance information, approximately $57.7 \%$ of the cases was Plasmodium vivax, followed by Plasmodium falciparum (40\%) and mixed infection of $P$. falciparum and $P$. vivax $(2.3 \%)$ in 2015 . In the same year, malaria mortality rate was 0.03 deaths per 100,000 . However, malaria morbidity rate, defined as annual parasite incidence which is the number of cases per 1000 population, was 4.9 based on the progress report for Lao PDR's Sustainable Development Goal 3.3.4a [3]. Recently, a study showed that asymptomatic malaria is a concern in Southeast Asia [4]. National malaria surveillance systems in this region rely on passive case detection that could not investigate asymptomatic malaria [5]. On the other hand, two province-wide community-based surveys targeting villagers with or without symptoms revealed that at least $95 \%$ of the cases were asymptomatic malaria [6, 7]. Common cases reported are among adults working or living in the forest areas to seek for the source of their livelihood [8]. Due to both the terrain and remoteness, most cases involve farmers and forest workers exposing them to outdoor biting vectors [9].

The military is one of the mobile population risk groups that work in forest and border areas where transmission intensity of malaria is high [10-12]. They comprise 2 to $3 \%$ of the annual malaria cases in Lao PDR. A community-based study was conducted in six villages in Attapeu province which showed that soldiers are significantly more likely to have malaria infection, compared to other villagers [6]. Studies that were conducted with the military in other countries reported that diagnosis and treatment are often delayed because of barriers to accessing health services which can contribute to prolonging infectivity, increasing drug resistance, and promoting diseases transmission $[13,14]$. No study has been conducted in Lao PDR which explores the burden of malaria and preventive and treatment behavior among military personnel. Therefore, information is lacking to guide and implement effective control strategies for this risk group.

This study aimed to (1) determine prevalence of malaria infection and (2) assess knowledge, perception, and preventive and treatment behavior regarding malaria among military personnel in Champasak and Attapeu provinces in Lao PDR.

\section{Materials and methods Study design}

In this study, a combination of quantitative (cross sectional study) and qualitative methods was used. The quantitative method included questionnaire-based interview, body temperature measurement, and malaria testing through rapid diagnostic test (RDT) and polymerase chain reaction (PCR) assay. The method aimed to examine knowledge, belief, preventive measures pertaining to malaria, and prevalence of infection. The qualitative method included focus group discussions (FGDs) and in-depth interviews (IDIs). The method aimed to explore perception and behavior.

\section{Study sites}

The two southernmost provinces, Champasak and Attapeu, were selected. Study districts were purposely selected by the Military Health Office using the following criteria: (1) presence of military camps in the national borders, and (2) high endemicity of malaria. These were Pathoumphone, Khong, Soukhouma, and Mounlapamok (four of ten) districts of Champasak province and Sanamxay (one of five) district of Attapeu province (Fig. 1). All the nine military camps in these districts were included in the study. Fieldwork was done in September while laboratory works were conducted in October 2017.

\section{Study participants}

A month prior to data collection, an invitation notice was posted in the target military camps inviting military personnel to join the study. Those who came on the day of data collection were oriented about the study including the inclusion criteria and data collection procedures, and that the military personnel were free to participate in the study. Military personnel who worked in the camps are at least 18 years old, and gave consent to underwent quantitative data collection. Out of the 351 military personnel, 313 were eligible for the study. Three hundred thirteen participants in quantitative study were asked to join in qualitative data collection referring to malaria episode. Among those, only 49 participants were able to wait and voluntarily join in either FGDs or IDIs.

\section{Quantitative data collection}

Five surveyors (one medical officer from the health sector and four medical officers from the military sector) were recruited for data collection. They were explained about the study objectives and underwent training on data collection procedures, blood sampling method, and ethical issues. The survey questionnaire included closed- and open- 


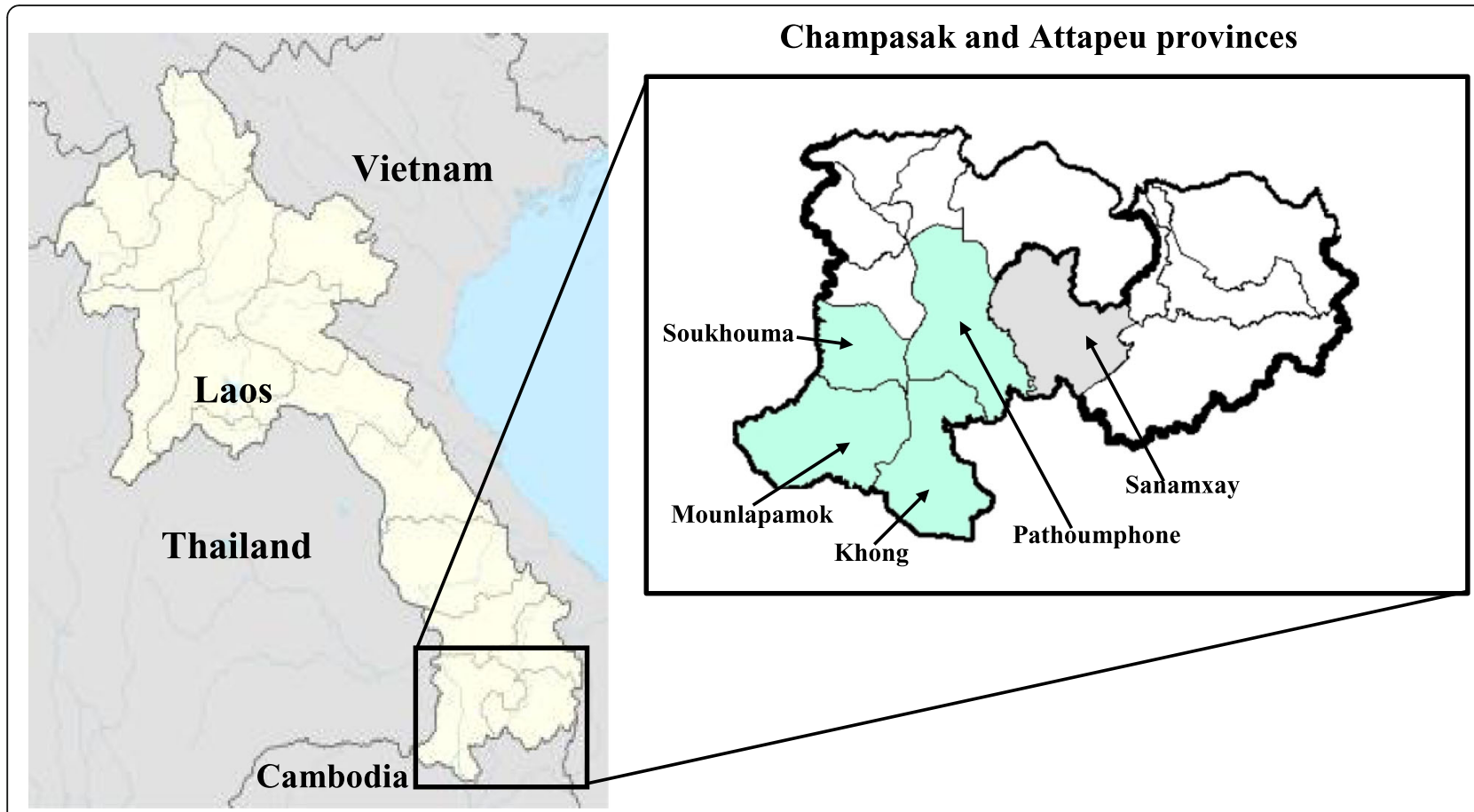

Fig 1 Map of the study sites in Soukhouma, Mounlapamok, Khong, Pathoumphone districts of Champasak province and Sanamxay district of Attapeu, Lao PDR

ended questions and consisted of four sections. The background characteristics section included questions on age, marital status, ethnic group affiliation, education level, monthly income, duration of work in remote forest areas, and previous malaria episodes in the past 1 year which obtained from all self-reported malaria. The knowledge section included questions on symptoms, transmission route, vector and breeding site, and preventive measures. The prevention section asked about routine preventive practices when participants work in forest areas. The beliefs section was on beliefs of malaria such as malaria being preventable, curable, possible cause of illness, or death. The questionnaire was pilot-tested among five military personnel in Vientiane, the capital city of Lao PDR. Minor modifications of the questionnaire were made to ensure that the words used were understandable and acceptable.

The interviews were conducted in a place where participants felt relaxed and confident to openly discuss the interview questions, mostly outside their camps. The interviews lasted about 40 to $45 \mathrm{~min}$. Twenty-five interviews were excluded due to incompleteness of the answers. The remaining 288 (92\%) were included in the analysis. Only 274 out of the 288 respondents were assessed for knowledge as 14 participants did not know about malaria.

\section{Plasmodium infection determination}

Two surveyors and three to four medical personnel from their respective camps assisted in Plasmodium infection determination. After the questionnaire-based interview, temperature was taken and $0.2-\mathrm{ml}$ blood samples were collected from the participants. Two diagnostic methods were used to detect Plasmodium infections in blood samples. The participants were detected on-site for malaria using RDT (SD Bioline Ag Pf/Pv, Standard Diagnostics, Inc., Gyeonggi-do, Republic of Korea). Blood samples were collected on filter papers (Whatman FTA Classic Cards, GE Health care Life Science, UK) for PCR analysis. All blood samples were analyzed using PCR method performed in Institut Pasteur du Laos. Asymptomatic infection was defined as Plasmodium infection detected in participants who presented with body temperature $\leq 37.5{ }^{\circ} \mathrm{C}$ and absence of any clinical symptoms of malaria at the time of blood sampling $[15,16]$, but tested positive by PCR. Those who had fever or tested positive in RDT during data collection was either referred or brought to the military hospital. The names of those who tested positive in PCR were forwarded to the Military Health Office which facilitated treatment for them.

\section{Laboratory procedures}

In accordance with the manufacturer's instructions, deoxyribonucleic acid (DNA) was extracted from dried blood spot on the filter papers with a QLAamp DNA Mini Kit (Qiagen, Hilden, Germany). The extracted DNA was eluted with $50 \mu \mathrm{L}$ of elution buffer in the kit and preserved at $-30{ }^{\circ} \mathrm{C}$. A real-time nested PCR was 
performed using a primer set to identify malaria parasite infection $[17,18]$. A universal primer set was use in the primary real-time PCR to amplify the partial cytochrome $b$ gene on the mitochondrial genome of malaria parasites. In the secondary real-time PCR, P. falciparum and $P$. vivax were detected using specific primer sets. The real-time PCR was performed using SsoAdvanced ${ }^{\mathrm{Tm}}$ Uni- $^{-}$ versal SYBR ${ }^{\bullet}$ Green Supermix (Bio-Rad Laboratory, Inc., USA) using $2 \mu \mathrm{L}$ of the extracted DNA as a template, which was equivalent to 1.2 to $1.6 \mu \mathrm{L}$ of whole blood. The primary PCR product was diluted 25 times with PCR-grade water, and $2 \mu \mathrm{L}$ of the diluted primary PCR product was used as a template for the secondary real-time PCR. Serial diluted recombinant plasmid DNAs containing the cytochrome $b$ region of $P$. falciparum and $P$. vivax were used as positive control for each assay, and PCR-grade water was used as negative control. A sample was considered negative if there was no indicated line increase in the $\mathrm{SYBR}^{\circ}$ Green (fluorescent) signal after 35 cycles. When positive results were obtained at least twice, the sample was considered positive for Plasmodium DNA [6].

\section{Qualitative data collection}

After the questionnaire-based interview, blood sampling, and temperature measurements, participants were invited to participate in IDI or FGD in the same or the following day. IDIs and FGDs were conducted in Lao language. The IDI and FGD guides comprise of questions related to participants' views about malaria, risks related to their living and working conditions, information about their health, their care-seeking and treatment behavior when getting malaria, preventive measure use for malaria in the forest, and sources of malaria information. Proceedings were recorded using a digital voice recorder with the participants' permission. Most of the FGDs and IDIs were carried out outside their camps. Immediately after the interviews, all were invited and selected referring to malaria episode. Those who gave consent had the option to participate in either FGD or IDI. Four to five participants joined the FGDs while 17 participants participated in IDIs. Seven FGDs with 32 participants and 17 IDIs were performed. The FGDs and IDIs lasted between 35 and $50 \mathrm{~min}$.

\section{Quantitative data analysis}

Data were double entered into Microsoft Excel version 2016. Incorrect entries were examined and verified against the original forms. The infection of malaria, prevalence, knowledge, and believe regarding malaria were analyzed and presented using frequencies. The participants' age, monthly income, and duration of work in the current military camp were treated as continuous variables and summarized as medians with interquartile range (IQR). Proportion and 95\% confidence interval (CI) of military infected with malaria using PCR was calculated. Fisher's exact test and Mann-Whitney $U$ test were used to assess the associations between malaria infection status determined by PCR assay and respondent's general information (age, gender, ethnicity, education, marital status, monthly income, and duration of work in the current military camp) and use of a prevention measure. A $p$ value $<0.05$ was considered statistically significant. These analyses were performed by SPSS version 23 .

\section{Qualitative data analysis}

Transcriptions of the IDIs and FGDs were done by the first author (PV). The transcripts were analyzed using content analysis, which is a stepwise analytical process, focusing on description and interpretation of underlying meanings of the text [19]. In order to minimize misinterpretations, interview notes were used side-by-side. Words, phrases, and sentences as a part of text in the transcript were used to develop codes. The codes that reflected the core meanings of the interview text were identified, and grouped into subcategories, categories, and themes. Data analysis was supervised and validated by the sixth author (JK) who is an expert on qualitative data analysis.

\section{Results}

\section{Background characteristics of the participants}

The median age of participants was 28 years and median work duration in forest areas was 4 years (Table 1). Majority of the participants (98.6\%) were men. The educational attainment was mostly high school or lower (81.9\%). Approximately less than half (45.5\%) of the participants were married. Approximately one-third (33.7\%) reported to experience a malaria episode at least once in the past year. Among almost all the participants (98.3\%), their body temperatures were equal to or less than $37.5^{\circ} \mathrm{C}$ during the survey. Among the 35 participants who tested positive by PCR, 34 (97.1\%) did not have a fever greater than $37.5^{\circ} \mathrm{C}$.

When comparing these characteristics between the positive and negative groups as detected by PCR, the working duration was significantly longer in the positive group than in the negative group $(p=0.030)$. For other characteristics, there were no significant differences between the two.

\section{Prevalence of malaria infection}

PCRs detected malaria parasitemia in 35 of 313 blood samples (11.2\%, 95\% CI 7.7 to 14.7) (Table 2). The species distribution was $P$. falciparum mono-infection at $1.3 \%$ (4/313), P. vivax mono-infection at 9.3\% (29/313), and $P$. falciparum and $P$. vivax mixed infections at $0.6 \%(2 / 313)$. RDTs identified only three $P$. vivax mono-infection, six P. falciparum mono-infection, and one P. falciparum and $P$. vivax mixed infection. 
Table 1 Characteristics of military personnel

\begin{tabular}{|c|c|c|c|c|c|c|c|}
\hline \multirow[t]{2}{*}{ Characteristics } & \multicolumn{2}{|l|}{ Total } & \multicolumn{2}{|c|}{ Positive } & \multicolumn{2}{|c|}{ Negative } & \multirow[t]{2}{*}{$p$ value } \\
\hline & $n=288$ & $\%$ & $n=35$ & $\%$ & $n=253$ & $\%$ & \\
\hline Age (years), median (IQR) & \multicolumn{2}{|c|}{28 (24 to 34$)$} & \multicolumn{2}{|c|}{27 (24 to 40$)$} & \multicolumn{2}{|c|}{28 (24 to 34$)$} & 0.455 \\
\hline Monthly income (USD), median (IQR) & \multicolumn{2}{|c|}{$151.9(102.3$ to 188.4$)$} & \multicolumn{2}{|c|}{151.9 (99.4 to 187.0$)$} & \multicolumn{2}{|c|}{151.9 (102.3 to 188.4$)$} & 0.593 \\
\hline Work in forest areas (years), median (IQR) & \multicolumn{2}{|c|}{$4(2$ to 7$)$} & \multicolumn{2}{|c|}{$5(2$ to 10$)$} & \multicolumn{2}{|c|}{3 (2 to 7 ) } & 0.030 \\
\hline \multicolumn{8}{|l|}{ Gender } \\
\hline Male & 284 & 98.6 & 35 & 100.0 & 249 & 98.6 & 1.000 \\
\hline Female & 4 & 1.4 & 0 & 0.0 & 4 & 1.4 & \\
\hline \multicolumn{8}{|l|}{ Ethnicity } \\
\hline Lao ethnic & 252 & 87.5 & 29 & 82.9 & 223 & 88.1 & 0.411 \\
\hline Other ethnic & 36 & 12.5 & 6 & 17.1 & 30 & 11.9 & \\
\hline \multicolumn{8}{|l|}{ Education } \\
\hline$\leq$ High school & 236 & 81.9 & 29 & 82.9 & 207 & 81.8 & 1.000 \\
\hline > High school & 52 & 18.1 & 6 & 17.1 & 46 & 18.2 & \\
\hline \multicolumn{8}{|l|}{ Marital status } \\
\hline Single & 157 & 54.5 & 22 & 62.9 & 135 & 53.4 & 0.366 \\
\hline Married & 131 & 45.5 & 13 & 37.1 & 118 & 46.6 & \\
\hline \multicolumn{8}{|l|}{ Episode of malaria (times) } \\
\hline Never & 191 & 66.3 & 21 & 60.0 & 170 & 67.2 & 0.671 \\
\hline 1 or more & 97 & 33.7 & 14 & 40.0 & 83 & 32.8 & \\
\hline \multicolumn{8}{|l|}{ Body temperature $\left({ }^{\circ} \mathrm{C}\right)$} \\
\hline$\leq 37.5$ & 283 & 98.3 & 34 & 97.1 & 249 & 98.4 & 0.479 \\
\hline$>37.5$ & 5 & 1.7 & 1 & 2.9 & 4 & 1.6 & \\
\hline \multicolumn{8}{|l|}{ Provinces } \\
\hline Champasak & 227 & 78.8 & 28 & 80.0 & 199 & 78.7 & 0.528 \\
\hline Attapeu & 61 & 21.2 & 7 & 20.0 & 54 & 21.3 & \\
\hline
\end{tabular}

\section{Knowledge on malaria}

Only $47.4 \%$ of the participants knew that malaria is transmitted by an Anopheles mosquito bite (Table 3). The participants believed that malaria is transmitted by drinking stream water from the forest (60.9\%). Fewer participants also believed that malaria is transmitted through coughing/sneezing $(28.5 \%)$ or by flies (25.5\%). In contrast, participants knew about abnormal health conditions that are considered malaria symptoms: more than $80 \%$ of the participants knew that

Table 2 Distribution of malaria infection according to species among those who tested positive by PCR $(n=35)$ and RDT $(n=10)$

\begin{tabular}{lll}
\hline Type of malaria infection & $P C R$ & RDT \\
& $n(\%)$ & $n(\%)$ \\
\hline P. vivax mono-infection & $29(82.9)$ & $3(30.0)$ \\
P. falciparum mono-infection & $4(11.4)$ & $6(60.0)$ \\
P. falciparum and $P$. vivax mixed-infection & $2(5.7)$ & $1(10.0)$ \\
\hline
\end{tabular}

Table 3 Knowledge on transmission routes and symptoms of malaria among study participants $(n=274)$

\begin{tabular}{llll}
\hline Knowledge & Yes & No & Do not know \\
& $n(\%)$ & $n(\%)$ & $n(\%)$ \\
\hline Malaria transmission route & & & \\
Anopheles mosquito biting & $130(47.4)$ & $29(10.6)$ & $115(42.0)$ \\
Drinking stream water in forest & $167(60.9)$ & $46(16.8)$ & $61(22.3)$ \\
Cough or sneeze & $78(28.5)$ & $102(37.2)$ & $94(34.3)$ \\
Flies & $70(25.5)$ & $100(36.5)$ & $104(38.0)$ \\
Spirit & $19(6.9)$ & $172(62.8)$ & $115(42.0)$ \\
Malaria symptoms & & & \\
Fever & $242(88.3)$ & $7(2.6)$ & $25(9.1)$ \\
Headache & $236(86.1)$ & $9(3.3)$ & $29(10.6)$ \\
Body pains & $235(85.8)$ & $7(2.6)$ & $32(11.7)$ \\
Chills & $234(85.4)$ & $10(3.6)$ & $30(10.9)$ \\
Weak/poor appetite & $173(63.1)$ & $51(18.6)$ & $50(18.2)$ \\
Vomiting & $163(59.5)$ & $44(16.1)$ & $67(24.5)$ \\
\hline
\end{tabular}


fever, headache, body pains, and chills are symptoms of malaria.

\section{Beliefs about malaria}

Most of the participants (93.8\%) showed fear of contracting malaria (Table 4). Most of the participants $(92.0 \%)$ believed that working in the forest puts them at risk of acquiring malaria. More than $80 \%$ of participants also believed that malaria is preventable, curable, but also can cause illness and death.

\section{Preventive measures}

Almost all participants (99.0\%) used a bed net (Table 5). Some of them $(n=80)$ owned LLIN. A few numbers of participants $(n=31)$ used hammock nets. Most of the participants (91.3\%) used mosquito repellent. Fewer participants used wood/plant smoke (68.4\%) and mosquito coil $(87.8 \%)$ when they stayed in a forest area. About two-thirds of the participants (73.6\%) took drug prophylaxis distributed in their camps. When comparing these preventive measures between positive and negative groups, drug prophylaxis was significantly associated with malaria infection $(p=0.039)$. There were no statistically significant associations observed between malaria infection and the other preventive measures.

\section{Content analysis on qualitative data}

The findings are presented according to the five themes that have emerged: (1) direct and indirect effects of malaria, (2) perception on malaria, (3) self-care, (4) health service delivery, and (5) access and use of preventive equipment (Table 6).

\section{Direct and indirect effects of malaria}

Malaria was recognized as the second most common health problem next to dengue fever. Majority of the participants recognized that $P$. vivax is common among military personnel and that malaria is a cause of their hospitalization. Upon hospitalization of the respondents or their colleagues, they ask the medical personnel about

Table 4 Beliefs on malaria infection among study participants $(n=288)$

\begin{tabular}{llll}
\hline & Yes & No & Do not know \\
& $n(\%)$ & $n(\%)$ & $n(\%)$ \\
\hline Fear of getting malaria & $270(93.8)$ & $11(3.8)$ & $7(2.4)$ \\
$\begin{array}{l}\text { Working in forest areas is } \\
\text { risk acquiring malaria }\end{array}$ & $265(92.0)$ & $8(2.8)$ & $15(5.2)$ \\
Malaria is preventable & $260(90.3)$ & $4(1.4)$ & $24(8.3)$ \\
Malaria is curable & $256(88.9)$ & $5(1.7)$ & $27(9.4)$ \\
Malaria causes of illness & $256(88.9)$ & $11(3.8)$ & $21(7.3)$ \\
Malaria can cause of death & $242(84.0)$ & $13(4.5)$ & $33(11.5)$ \\
\hline
\end{tabular}

Table 5 Preventive measure of malaria among study participants $(n=288)$

\begin{tabular}{|c|c|c|c|c|}
\hline \multirow[t]{2}{*}{$\begin{array}{l}\text { Prevention } \\
\text { practices }\end{array}$} & $\begin{array}{l}\text { Total } \\
n=288\end{array}$ & $\begin{array}{l}\text { Positive } \\
n=35\end{array}$ & $\begin{array}{l}\text { Negative } \\
n=253\end{array}$ & \multirow[t]{2}{*}{$p$ value $^{a}$} \\
\hline & $n(\%)$ & $n(\%)$ & $n(\%)$ & \\
\hline \multicolumn{4}{|l|}{ Bed net use } & \multirow[t]{3}{*}{1.000} \\
\hline Yes & $285(99.0)$ & $35(100.0)$ & $250(98.8)$ & \\
\hline No & $3(1.0)$ & $0(0.0)$ & $3(1.2)$ & \\
\hline \multicolumn{4}{|c|}{ Ordinary bed net } & \multirow[t]{3}{*}{0.837} \\
\hline Yes & $215(74.7)$ & $27(77.1)$ & $188(74.3)$ & \\
\hline No & $73(25.3)$ & $8(22.9)$ & $65(25.7)$ & \\
\hline \multicolumn{4}{|l|}{ LLINS } & \multirow[t]{3}{*}{0.226} \\
\hline Yes & $80(27.8)$ & $13(37.1)$ & $67(26.5)$ & \\
\hline No & $208(72.2)$ & $22(62.9)$ & $186(73.5)$ & \\
\hline \multicolumn{4}{|c|}{ Hammock net } & \multirow[t]{3}{*}{1.000} \\
\hline Yes & $31(10.8)$ & $3(8.6)$ & $28(11.1)$ & \\
\hline No & $257(89.2)$ & $32(91.4)$ & $225(88.9)$ & \\
\hline \multicolumn{4}{|c|}{ Wearing long uniform } & \multirow[t]{3}{*}{0.713} \\
\hline Yes & $269(93.4)$ & $32(91.4)$ & $237(93.7)$ & \\
\hline No & $19(6.6)$ & $3(8.6)$ & $16(6.3)$ & \\
\hline \multicolumn{4}{|c|}{ Repellents use } & \multirow[t]{3}{*}{0.750} \\
\hline Yes & $263(91.3)$ & $33(94.3)$ & $230(90.9)$ & \\
\hline No & $25(8.7)$ & $2(5.7)$ & $23(9.1)$ & \\
\hline \multicolumn{4}{|c|}{ Mosquito coil use } & \multirow[t]{3}{*}{0.782} \\
\hline Yes & $253(87.8)$ & $32(31.4)$ & $221(87.4)$ & \\
\hline No & $35(12.2)$ & $3(8.6)$ & $32(12.6)$ & \\
\hline \multicolumn{4}{|c|}{ Wood/plant smoke use } & \multirow[t]{3}{*}{0.702} \\
\hline Yes & $197(68.4)$ & $23(65.7)$ & $174(68.8)$ & \\
\hline No & $91(31.6)$ & $12(34.3)$ & $79(31.2)$ & \\
\hline \multicolumn{4}{|c|}{ Taking drug prophylaxis } & \multirow[t]{3}{*}{0.039} \\
\hline Yes & $212(73.6)$ & $31(88.6)$ & $181(71.5)$ & \\
\hline No & $76(26.4)$ & $4(11.6)$ & $72(28.5)$ & \\
\hline
\end{tabular}

${ }^{\text {aFisher's exact test }}$

their sickness hence they are able to identify the cause of their condition. They have also observed the symptoms among their sick colleagues. This information is shared in their camps. Participants commented that it is not easy to protect themselves from malaria infection. One participant who had the infection narrated how this occurred.

The common disease among the military is malaria. I cannot avoid getting infected. In 2014, the doctor told me that I got mixed-infection with P. falciparum and $P$. vivax, then I got the infection again in 2015 and 2016 with $P$. falciparum, and in 2017 with $P$. vivax. [22-32-year-old military, in FGD 3, Khong district].

Not only themselves but also their family members suffered from malaria. Participants who were living with their 
Table 6 Themes, categories, and sub-categories structure

\begin{tabular}{|c|c|c|}
\hline Themes & Categories & Sub-categories \\
\hline 1. Direct and indirect effects of malaria & Common health problem & $\begin{array}{l}\text { Dengue fever } \\
\text { Malaria episode } \\
\text { Health problems in the family } \\
\text { Other diseases }\end{array}$ \\
\hline 2. Perception of malaria & $\begin{array}{l}\text { Information sources } \\
\text { Lack of knowledge } \\
\text { Understanding of malaria }\end{array}$ & $\begin{array}{l}\text { Dangerous areas } \\
\text { Cause of malaria } \\
\text { Fear of malaria infection }\end{array}$ \\
\hline 3. Self-care & $\begin{array}{l}\text { Preventive behaviors } \\
\text { Purchase of personal preventive tools } \\
\text { Self-medication } \\
\text { Seeking care }\end{array}$ & $\begin{array}{l}\text { Preventive measures } \\
\text { Good practice } \\
\text { Emphasizing on repellent and LLIN use }\end{array}$ \\
\hline 4. Health service delivery & $\begin{array}{l}\text { Process of making decision } \\
\text { Health care services }\end{array}$ & $\begin{array}{l}\text { Field services } \\
\text { Referral patients } \\
\text { Health facilities which hey access when they get sick }\end{array}$ \\
\hline 5. Access and use of preventive equipment & $\begin{array}{l}\text { Insufficiency of preventive tools } \\
\text { Lack of diagnostic kits and anti-malaria } \\
\text { Difficulty in accessing health facilities } \\
\text { Difficulty in using bed nets } \\
\text { Exposure to malaria }\end{array}$ & \\
\hline
\end{tabular}

family members reported that malaria affected many aspects of their lives, especially on finances. Despite that the malaria treatment is free at hospitals, participants said that expenses for medicines other than antimalarials, and other daily medical expenses are not fully covered. Therefore, many participants spent their families' money or needed to borrow money from their relatives.

\section{Perception of malaria}

The main sources of information about malaria were television, colleagues in their camp, and the people in the nearby village. Many participants are concerned since others in the military community have been seriously infected with malaria. Additionally, they have expressed concern that they were working in malaria high-risk areas. It was hard for the participants to differentiate malaria and dengue fever as the symptoms of these diseases were similar. Many explained that malaria transmission was through a mosquito bite. Among those who were infected, they confidently said that an Anopheles mosquito bite is the cause. Many participants believed that being weak or not having enough rest make them at risk of contracting malaria. Some said that people with good physical conditions or a healthy person could not have malaria. Some participants believe that drinking unclean water from breeding sites is the cause, especially if it is contaminated with mosquito eggs, as this military elaborated:

There are many streams and ponds where mosquito reproduction happens. When we drink water, especially if it is contaminated with mosquito eggs, then we get infected. [22-33-year-old, military in FGD 1, Pathoumphone district].

\section{Self-care}

Most of the participants relied on preventive tools such as ordinary bed nets, mosquito repellents, and mosquito coils to protect themselves from mosquitoes. Some of them had used LLINs or insecticide-treated bed nets (ITNs). They emphasized that LLINs and repellents are necessary and there should not be any shortage for these in military camps in forests. In addition, some of the participants had to buy their own preventive tools at local markets. Chloroquine (CQ) was distributed for malaria prophylaxis only in Champasak province.

Participants noted that self-medication was their first response in the forest because of the limited access to treatment services. Antibiotics and painkillers were commonly used before referring a patient to a healthcare facility, but for some, traditional/herbal medicine was used as an alternative. Most of the participants reported that they used medicines that they brought or provided by the camp. If they do not feel better in 2 to 3 days, they would consult the health advisor or request a colleague to help them to seek medical care. One young man explained his ill-health condition:

I had fever in the forest and I was recommended not to take anti-malaria pills as it will influence the results of the diagnostic test. I took the painkillers like Paracetamol and just waited. It took me two to three days from the hilly forest area to meet the camp health advisor. I was then sent to a health center. [28-year-old, military, IDI 17, Sanamxay district]. 


\section{Health service delivery}

Based on the participant's discussions, military body and the military medical team have established healthcare services. General healthcare services and essential medicines are provided to the military personnel except malaria test kits and antimalarial medicine. In addition, a referral system has been launched to provide early advanced medical care. Although the healthcare services are in place, these are not fully established in all camps. When a patient has to be referred, some had to wait for 2 days to a week. There were no on-site malaria diagnostic and treatment services, despite that they have to work 2 to 3 months in the remote forest areas. When somebody is ill, the existing healthcare services are used. Almost all participants said that they first seek consult to their camp health advisor but the decision to refer the military manifesting clinical symptoms to a healthcare facility is made by the military team in which the ill patient is a member. Those who had not sought treatment said that they live very far from health facilities or they did not know where to get treatment. Those who were referred to community health centres and district hospitals said that there are enough medical supplies in these facilities.

\section{Access and use of protective equipment}

Basic preventive tools such as ordinary bed net, repellents, and mosquito coils were distributed free of charge in the camps. In addition, LLINs came from local health offices. However, there is still a lack of preventive equipment which limited the participants' ability to protect themselves against mosquitoes. Some participants used repellents, anti-mosquito lotions, coils and hammock nets, or burned fire wood to create smoke while doing outreach inspection outside the camps at night. Inadequate distribution of LLINs emerged in some focus groups. One military personnel said:

We received LLINs in 2016 but only two nets for our group were provided. Only two persons can use it at a time. We need nets for everyone in the camp.

[22-33-year-old, military in FGD 1, Pathoumphone district].

Several participants said that while traveling in the evening, when mosquitoes are active, they do not to protect themselves from mosquito bites as there is a lack of space in the forest to hang the bed nets, difficulty in using these particularly during the rainy season, and inability to use these during security inspections. It was often said that participants had to buy preventive tools at local markets. Participants also argued that not only preventive tools should be provided. There should also be on-site diagnostics tests and antimalaria drugs. They added that they had to wait for several days for them to be treated in the camps. In addition, drug prophylaxis for malaria was also requested from the interviewer.

\section{Discussion}

In the present study, the prevalence of malaria infection determined by PCR was $11.2 \%$ (35/313). Most of the infections $(82.9 \%)$ were $P$. vivax mono-infections and almost all the infections were asymptomatic (97.1\%). The finding suggests that the military in the study sites are at risk of $P$. vivax infection. This finding is similar to that from a community-based study conducted in the endemic villages in three districts of Attapeu province including Sanamxay district where the present study was also conducted. The study reported that the prevalence of malaria infection was $6.6 \%$, and $P$. vivax mono-infection and asymptomatic infection account for $87.2 \%$ and $97.9 \%$ of the total infection, respectively. Evidence suggests that individuals with asymptomatic, low-density malaria infection can contribute to local transmission [6, 20]. Therefore, without addressing malaria among the military in southern provinces, it would be impossible to achieve the target of Lao National Malaria Control and Elimination Program: eliminating malaria in the country by 2030 .

Most of the infections were due to $P$. vivax as revealed in the present study that was similar to a study among communities in Nong district, Savannakhet province, Lao PDR [7]. It requires to have a test of glucose-6-phosphate dehydrogenase (G6PD) deficiency before treatment of each vivax malaria patient by primaquine therapy. G6PD test and primaquine is available in provincial and district hospitals [6]. A 14-day course of primaquine was recommended to complete radical cure of vivax malaria, but this drug can cause a serious side effect, e.g., from mild to severe hemolysis in patients with glucose-6-phosphate dehydrogenase (G6PD) deficiency [21]. The patients that were infected by vivax malaria had moderate levels of serious G6PD deficiency among Lao population was reported [22]. Most of the vivax infection patients reside in remote areas, which are far from the hospitals. Additionally, health centers are not allowed to prescribe primaquine (as indicated in the guidelines) [6]. The limited access to G6PD test and primaquine in rural areas may contribute to delaying elimination of malaria. These problems can only be addressed by improving the policies, guidelines, and the facilities.

In the present study, PCR was used to identify four falciparum mono-infections, whereas RDT was used to identify six falciparum mono-infections. The difference might be due to false positive results of RDT, because the sensitivity and specificity of PCR for P. falciparum is higher than those of RDT [23], and because the histidine rich protein 2 (HRP-2), which is a target antigen for detecting P. falciparum, can persist for 28 days in peripheral blood even after effective treatment [24]. 
The present study showed that, although most participants understood the connection between malaria and mosquito, they did not necessarily know the name of malaria vector mosquitoes (i.e., Anopheles). The fact that people in a malaria endemic area understand the connection between malaria and mosquito was also reported in a recent Lao study that was conducted with general population in Nong district: The study reported that $96.8 \%$ of the participants understood the connection between malaria and mosquito [25].

Adequate knowledge and perception of malaria is important for its prevention [26, 27]. It also allows timely diagnosis and improves treatment seeking behavior [28]. The participants of the present study acknowledged the connection between the forest and malaria. However, many participants believed that drinking stream water in forests can cause malaria. A study that were conducted in Nong district showed the villagers described proper knowledge of the prominent malaria symptom, such as fever with chills and beliefs that malaria is a cause of severe illness and death [29]. The finding of the present study was consistent with that of the Nong study. The participants of the present study also believed that not only mosquito bites but also drinking unclean water can transmit malaria. This finding is also compliant with the Nong studies [25, 29]. These studies show that villagers make a link between malaria and poor hygiene, as well as via a mosquito bite. Additionally, the participants perceived that having good physical conditions or being healthy protects them from contracting malaria. Also, this result was similar with the Nong district study which revealed that some believe that cleaner persons will not have malaria [29]. This erroneous perception of malaria transmission may lead to incorrect preventive practices. For instance, a study in northern Ghana showed that people who have limited malaria knowledge including the role of vector were less likely to use ITNs [30]. In Nigeria, caregivers of children under-five who did not know what causes malaria and did not know methods to prevent malaria were less likely to use ITNs, even though they owned one [31]. Providing health education to the military community can promote correct understanding of malaria transmission route. It can also enhance their health concern, treatment-seeking behavior, and preventive practices. This would help them improve preventive practices for malaria. Furthermore, the participants who were dispatched to high-risk areas of malaria infection should know malaria, especially its symptoms. This allows them to recognize malaria infection and seek early treatment.

The study participants used a variety of preventive measures to prevent mosquito bites and three-fourths of them owned ordinary bed nets. A study in a neighboring country found out that even if untreated bed net could prevent malaria in village community, it was not suitable for persons who work in forest areas [32]. A study in India showed that malaria prevalence among people who used insecticide-treated nets was lower than those who used untreated bed net in low endemic and high endemic areas [33]. Moreover, shifting malaria prevention activities to the use of LLINs significantly reduced malaria prevalence [34]. Also, in Lao PDR, malaria intervention activities which includes LLINs distribution to general population at risk succeeded in reducing the number of malaria cases by $92 \%$ in 2010 [2]. Evidence suggests that if LLINs would be widely distributed to military, then malaria prevalence among those assigned in forest areas would become lower.

When the participants worked and traveled overnight in the forest, they had some difficulty in using bed nets because of inappropriate location and they do not have proper sleeping areas. Among the participants, hammock net was familiar and suitable for them. However, only $10.8 \%$ of the participants owned a hammock net. Additionally, those who did not have hammock net consequently burned firewood to produce smoke that will prevent mosquito bites at night. In the Cambodian and Vietnam border, hammock net was popularly used among young and adult men when sleeping overnight outside their homes [35]. In the same manner, long-lasting insecticide hammock nets (LLIHs) were effective in reducing malaria vector bites and protecting forest workers in Cambodia [36]. Furthermore, LLIH was identified to be a feasible method in reducing the incidence of malaria in forest areas [37]. The participants of the present study often worked and slept outside their camps in the forest. Hence, LLIHs should be an additional preventive tool for the military who work for security inspections in Lao PDR.

At night, while on security inspection and before going to sleep, participants did not use bed nets. Furthermore, the results of the qualitative analysis revealed that mosquito repellent was often unavailable when necessary. This was because the military authority provided an inadequate supply of repellent lotion to the staff. The results of a systematic review showed that high level of protection by repellent was effective against Anopheles gambiae s.l. in the field in Tanzania. Moreover, repellents were effective in reducing malaria morbidity [38]. Also, distribution of highly effective repellents could prevent malaria infection [39]. Improvement and ensuring adequate repellent supply to military employed in forest areas could solve the issue of mosquito biting when the participants work during the active hours of the mosquito vectors.

The present study found that taking drug prophylaxis was significantly associated with malaria infection status. The association is likely to be confounded by third factors; i.e., factors other than the drug prophylaxis and 
malaria infection status. Especially the association could be due to confounding by indication. That is, participants for whom drug prophylaxis was prescribed are often at higher risk of malaria infection, compared to those for whom drug prophylaxis was not prescribed. Thus, participants who reported taking drug prophylaxis showed higher prevalence, compared to those who did not.

The present study showed that taking chloroquine prophylaxis was common among study participants. More attention should be paid to this practice, because inappropriate use of chloroquine can promote chloroquine resistance [40]. Although the present study did not examine how participants administered prophylaxis, there is a possibility of sub-optimal use of chloroquine among participants, as reported by a study in Africa [41]. Additionally, non-compliance with antimalarial drug regimen or receiving sub-optimal dose led to increased risk of treatment failure [42]. Poor adherence to antimalarial medication for uncomplicated malaria was observed among rural communities in Lao PDR [43]. Failure to monitor this concern would affect prevalence of antimalarial drug resistance [44].

Case management of malaria in military health service at forest areas was not effective. Recently, an initial cooperation between the military organization and the $\mathrm{Na}$ tional Malaria Control Program (NMCP) have begun which included training military doctors and medics with the current national guidelines such as case management and prevention strategies [10]. However, case management procedures should be improved. These procedures should be aligned with the national guidelines on malaria treatment. There should be appropriate diagnostic tool kits and antimalarial drug combination therapy (ACTs) as current first-line treatment as introduced by the National Program $[45,46]$ to improve early access to malaria diagnosis and treatment [15]. The NMCP should assist the medical teams in the military to ensure adequate supply of malaria diagnosis tools (RDTs) and ACTs [47] and skilled military healthcare workers through training on case management. The program should also assist in monitoring these interventions among the military. Malaria mobile teams or malaria posts were very helpful to reduce malaria prevalence among villages in remote areas in neighboring countries $[48,49]$. A regional malaria elimination program that was implemented in Eastern Myanmar showed that providing early diagnosis and effective treatment through community-based malaria posts substantially decreased village-level incidence of malaria [50]. It is possible to train camp military medical or military personnel and participate in malaria posts. They can cover catchment areas to improve early diagnosis and treatment.

The present study discovered some difficulty of receiving malaria diagnosis and treatment at the health facilities due to the lack of medical personnel and inadequate health facilities. There is a need to improve accessibility to healthcare services for malaria treatment in remote communities [29]. In addition, poor accessibility to health facilities by limited transportation and high-hilly road condition was a common problem in isolated remote villages preventing residents to receive timely malaria treatment [51].

The results identified major challenges in malaria control such as the lack of health information particularly about the causes and transmission route. The delay in seeking treatment may be due to the very remote location of the camps in hilly forests making self-medication as the best alternative among the participants [52]. Poor access to healthcare services for malaria treatment was due to long distance to the health facility as well as a high cost of transportation and referral [53]. The unavailability of RDTs and ACTs, and inadequate supply of appropriate protection tools such LLINs, repellents, anti-mosquito lotions, and coils is also a major challenge. On the other hand, the government provided intermittent and limited protective tools to this at risk population [54]. However, improving the cooperation with the Ministry of Health especially for malaria control is crucial to provide appropriate and updated information, technical support, and medical equipment. Other government agencies, such as the Ministry of Finance and Ministry of Welfare, should assist the military body in implementing sustainable interventions.

Data were only collected from military personnel who were available on the day of interview. The information of those who were absent or were in a health facility was not covered. Therefore, the present study might underestimate the prevalence of malaria infection. The camps recruited in the study were not randomly selected, and thus, the generalizability of this study's findings can be limited. Although the data collection assistants were trained, they were military technical medical officers. This may lead to an interviewer bias since the assistant may want to avoid a negative social image. The proportion of participants who knew the name of vector mosquitoes (Anopheles) might be overestimated, because when the question was asked, "Anopheles mosquito" was provided as one of the response options. Despite these, this study provided important information that can be used when designing services and information campaigns for the military in this particular context.

\section{Conclusion}

Military in forest border areas are at risk for malaria infection. The prevalence of malaria was $11.2 \%$ with $82.9 \%$ of which was Plasmodium vivax mono-infection. More than a half of the military in forest areas believed that malaria is transmitted by drinking stream water. Military practice self-medication using either antibiotics and painkillers or traditional medicine before referring patients to undergo malaria testing and treatment. Protective equipment such as LLINs and mosquito repellents 
were insufficient. There was also a lack of diagnostic and treatment services in the camps, and inaccessibility of health services. The NMCP and the military body should provide health education and widely distribute protective equipment. There should also be on-site malaria testing and case management to prevent delays in accessing appropriate medical care.

\section{Abbreviations}

ACTs: Antimalarial drug combination therapy; FGDs: Focus group discussions; IDIs: In-depth interviews; IQR: Interquartile range; LLIH: Long-lasting insecticide hammock net; LLIN: Long-lasting insecticide-treated net; NMCP: National Malaria Control Program; PCR: Polymerase chain reaction; RDT: Rapid diagnosis test

\section{Acknowledgements}

We thank the participants for spending their valuable time and sharing their experiences. We acknowledge the assistance of the General Logistic Department, Ministry of Defense and local military offices in Champasak and Attapeu provinces for approving the implementation of this study. Special thanks to Dr. Simone Nambanya and Dr. Viengxay Vanisaveth who supported and facilitated the conduct of the study, as well as SATREPS project staff who assisted in the laboratory activities.

\section{Funding}

Department of Global Health, School of Health Sciences, Faculty of Medicine, University of the Ryukyus, Japan and Japan International Cooperation Agency (JICA)/ Science and Technology Research Partnership for Sustainable Development program (SATREPS) project for "Development of Innovative Research Technique Epidemiology of Malaria and other Parasitic Diseases" supported the study.

\section{Availability of data and materials}

Raw data may be obtained from the corresponding author upon request.

\section{Authors' contributions}

PV was the principal investigator developed the research design, prepared data collection, supervised research assistants during data collection, carried out the analysis, and drafted the manuscript. DN, PS, ML, MI, JK, SKO, BH, PTB, and SKA assisted with the research design. MI supported the PCR analysis. DN, $\mathrm{JK}, \mathrm{PH}$, and $\mathrm{KP}$ offered critical comments in reviewing the manuscript. All the authors have read and approved the final manuscript.

\section{Ethics approval and consent to participate}

Ethical clearance was obtained from the National Ethics Committee for Health Research, Lao PDR (No. 073/NECHR), and from the Ethics Review Committee for Epidemiology Study, University of the Ryukyus (No. 1172). The permission to conduct the study was given by the General Logistic Department, the Ministry of Defense (No. 358), Lao PDR, and also from the camp leaders. The interviewers and moderator explained the purpose of the study and procedures in Laotian. The participants were also informed that their participation was voluntary, and that the results of the blood analyses and interviews would be treated in confidence. Moreover, they were informed that they can withdraw from the interview and discussion anytime without consequences.

\section{Consent for publication}

Not applicable.

\section{Competing interests}

The authors declare that they have no competing interests.

\section{Publisher's Note}

Springer Nature remains neutral with regard to jurisdictional claims in published maps and institutional affiliations.

\section{Author details}

'Department of Global Health, Graduate School of Health Sciences, University of the Ryukyus, 207 Uehara-cho, Okinawa 903-0215, Japan. ${ }^{2}$ Center of Malariology, Parasitology and Entomology, Ministry of Health, Vientiane, Lao PDR. ${ }^{3}$ SATREPS Project for Parasitic Diseases, Vientiane, Lao PDR. ${ }^{4}$ Military
Institute of Diseases Prevention, Department of Military Medical, Ministry of Defense, Vientiane, Lao PDR. ${ }^{5}$ Department of Tropical Medicine and Malaria Research Institute, National Center for Global Health and Medicine, 1-21-1

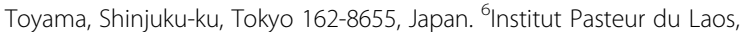
Ministry of Health, Vientiane, Lao PDR. ${ }^{7}$ Department of Environmental and Occupational Health, College of Public Health, University of the Philippines Manila, 625 Pedro Gil Street, Ermita, 1000 Manila, Philippines. ${ }^{8}$ Academic Affairs Division, University of Health Sciences, Vientiane, Lao PDR. ${ }^{9}$ Lao Tropical and Public Health Institute, Ministry of Health, Vientiane, Lao PDR.

Received: 25 October 2018 Accepted: 14 January 2019 Published online: 25 January 2019

\section{References}

1. Jorgensen P, Nambanya S, Gopinath D, Hongvanthong B, Luangphengsouk $\mathrm{K}$, Bell $\mathrm{D}$, et al. High heterogeneity in Plasmodium falciparum risk illustrates the need for detailed mapping to guide resource allocation: a new malaria risk map of the Lao People's Democratic Republic. Malar J. 2010;9:59.

2. Ministry of Health. Lao People's Democratic Republic. National strategic plan for malaria control and elimination 2016-2020. Lao People's Democratic Republic: Ministry of Health; 2016. http://www.malariafreemekong.org/2017/ 08/02/lao-pdr-2016-2020/. Accessed 25 Dec 2018.

3. United Nation. Lao People's Democratic Republic. Voluntary National Review on the Implementation of the 2030 Agenda for Sustainable Development. https://sustainabledevelopment.un.org/content/documents/19385Lao_ Final_VNR_19_June_2018_web.pdf. Accessed 28 Sept 2018.

4. Imwong M, Nguyen TN, Tripura R, Peto TJ, Lee SJ, Lwin KM, et al. The epidemiology of subclinical malaria infections in South-East Asia: findings from cross-sectional surveys in Thailand-Myanmar border areas, Cambodia, and Vietnam. Malar J. 2015;14:381.

5. Pirahmadi S, Zakeri S, Raeisi A. Absence of asymptomatic malaria infection in a cross-sectional study in Iranshahr district, Iran under elimination programmes. Iran J Parasitol. 2017;12:90-100.

6. Iwagami M, Keomalaphet $S$, Khattignavong P, Soundala P, Lorphachan L, Takahashi EM, et al. The detection of cryptic Plasmodium infection among villagers in Attapeu province, Lao PDR. PLoS Negl Trop Dis. 2017;11: e0006148.

7. Phommasone K, Adhikari B, Henriques G, Phongvongsa T, Phongmany P, Seidlein LV, et al. Asymptomatic Plasmodium infections in 18 villages of southern Savannakhet province, Lao PDR (Laos). Malar J. 2016;15:296.

8. United Nation Office Project Services. Malaria services for mobile migrant populations and forest-goers in Lao PDR. https://www.raifund.org/en/news/ malaria-services-mobile-migrant-populations-and-forest-goers-lao-pdr. Accessed 28 August 2018.

9. Tangena JAA, Thammavong P, Lindsay SW, Brey PT. Risk of exposure to potential vector mosquitoes for rural workers in northern Lao PDR. PLoS Negl Trop Dis. 2017;11:e0005802.

10. Kounnavong S, Gopinath D, Hongvanthong B, Khamkong C, Sichanthongthip O. Malaria elimination in Lao PDR: the challenges associated with population mobility. Infect Dis Poverty. 2017;6:81.

11. Wen S, Harvard KE, Gueye CS, Canavati SE, Chancellor A, Ahmed BN, et al. Targeting populations at higher risk for malaria: a survey of national malaria elimination programmes in the Asia Pacific. Malar J. 2016;15:271.

12. Manning JE, Satharath P, Gaywee J, Lopez MN, Lon C, Saunders DL. Fighting the good fight: the role of militaries in malaria elimination in Southeast Asia. Trends Parasitol. 2014;30:571-81.

13. Chretien JP, Blazes DL, Coldren RL, Lewis MD, Gaywee J, Kana K, et al. The importance of militaries from developing countries in global infectious disease surveillance. Bull World Health Organ. 2007:85:174-80.

14. Ho ZJM, Hwang YFJ, Lee JMV. Emerging and re-emerging infectious diseases: challenges and opportunities for militaries. Military Medical Research. 2014;1:21.

15. World Health Organization. Guidelines for the treatment of malaria third edition. Geneva: World Health Organization; 2015. http://www.who.int/ malaria/publications/atoz/9789241549127/en/. Accessed 28 Aug 2018.

16. Lindblade KA, Steinhardt L, Samuels A, Kachur SP, Slutsker L. The silent threat: asymptomatic parasitemia and malaria transmission. Expert Rev AntiInfect Ther. 2013;11:623-39.

17. Putaporntip C, Buppan P, Jongwutiwes S. Improved performance with saliva and urine as alternative DNA sources for malaria diagnosis by mitochondrial DNA-based PCR assays. Clin Microbiol Infect. 2011;17:1484-91. 
18. Tanizaki R, Ujiie M, Kato Y, Iwagami M, Hashimoto A, Kutsuna S, et al. First case of Plasmodium knowlesi infection in a Japanese traveller returning from Malaysia. Malar J. 2013;12:128.

19. Graneheim UH, Lundman B. Qualitative content analysis in nursing research: concepts, procedures and measures to achieve trustworthiness. Nurse Educ Today. 2004;24:105-12.

20. Iwagami M, Nakatsu M, Khattignavong P, Soundala P, Lorphachan L, Keomalaphet $\mathrm{S}$, et al. First case of human infection with Plasmodium knowlesi in Laos. PLoS Negl Trop Dis. 2018;12:e0006244.

21. World Health Organization. Control and elimination of Plasmodium vivax malaria: a technical brief. 2015. http://apps.who.int/iris/bitstream/handle/ 10665/181162/9789241509244_eng.pdf;jsessionid= 86582E75F22DE701E72C82F87B5F2BE5? sequence=1. Accessed 3 Jan 2018.

22. Lover AA, Dantzer E, Hongvanthong B, Chindavongsa K, Welty S, Reza T, et al. Prevalence and risk factors for asymptomatic malaria and genotyping of glucose 6-phosphate (G6PD) deficiencies in a vivax-predominant setting, Lao PDR: implications for sub-national elimination goals. Malar J. 2018;17:218.

23. Ranadive N, Kunene S, Darteh S, Ntshalintshali N, Nhlabathi N, Dlamini N, et al. Limitations of rapid diagnostic testing in patients with suspected malaria: a diagnostic accuracy evaluation from Swaziland, a low-endemicity country aiming for malaria elimination. Clin Infect Dis. 2017;64:1221-7.

24. Swarthout TD, Counihan H, Senga RKK, Broek IVD, et al. Paracheck-Pf ${ }^{\oplus}$ accuracy and recently treated Plasmodium falciparum infections: is there a risk of over-diagnosis? Malar J. 2007;6:58.

25. Adhikari B, Phommasone K, Pongvongsa T, Kommarasy P, Soundala X Henriques $\mathrm{G}$, et al. Factors associated with population coverage of targeted malaria elimination (TME) in southern Savannakhet Province, Lao PDR. Malar J. 2017:16:424.

26. Kimbi HK, Nkesa SB, Nyanga JLN, Sumbele IU, Atashili J, Atanga MBS Knowledge and perceptions towards malaria prevention among vulnerable groups in the Buea Health District, Cameroon. BMC Public Health. 2014;14:883.

27. Barja MR, Ncogo P, Nseng G, Morales MAS, Herrador Z, Berzosa P, et al. Caregivers' malaria knowledge, beliefs and attitudes, and related factors in the Bata district, Equatorial Guinea. PLoS One. 2016;11:e0168668.

28. Naing PA, Maung TM, Tripathy JP, Oo T, Wai KT, Thi A. Awareness of malaria and treatment-seeking behaviour among persons with acute undifferentiated fever in the endemic regions of Myanmar. Trop Med Health. 2017:45:31.

29. Adhikari B, Phommasone K, Kommarasy P, Soundala X, Souvanthong P, Pongvongsa $\mathrm{T}$, et al. Why do people participate in mass anti-malarial administration? Findings from a qualitative study in Nong District, Savannakhet Province, Lao PDR (Laos). Malar J. 2018;17:15.

30. Adongo PB, Kirkwood B, Kendall C. How local community knowledge about malaria affects insecticide-treated net use in northern Ghana. Tropical Med Int Health. 2005:10:366-78.

31. Arogundade ED, Adebayo SB, Anyanti J, Nwokolo E, Ladipo O, Ankomah A, et al. Relationship between care-givers' misconceptions and non-use of ITNs by under-five Nigerian children. Malar J. 2011;10:170.

32. Erhart A, Thang ND, Hung NQ, Toi LV, Hung LX, Tuy TQ, et al. Forest malaria in Vietnam: a challenge for control. Am J Trop Med Hyg. 2004;70:110-8.

33. Remoortel HV, Buck ED, Singhal M, Vandekerckhove P, Agarwal SP. Effectiveness of insecticide-treated and untreated nets to prevent malaria in India. Tropical Med Int Health. 2015;20:972-82.

34. Hetzel MW, Pulford J, Ura Y, Maiasa SJ, Tandrapah A, Tarongka N, et al. Insecticide-treated nets and malaria prevalence, Papua New Guinea, 20082014. Bull World Health Organ. 2017;95:695-705B.

35. Gryseels C, Grietens KP, Dierickx S, Xuan XN, Uk S, Tyrrell MB, et al. High mobility and low use of malaria preventive measures among the Jarai male youth along the Cambodia-Vietnam border. Am J Trop Med Hyg. 2015;93:810-8.

36. Sochantha T, Bortel WV, Savonnaroth S, Marcotty T, Speybroeck N, Coosemans M. Personal protection by long-lasting insecticidal hammocks against the bites of forest malaria vectors. Tropical Med Int Health. 2010;15: 336-41.

37. Thang ND, Erhart A, Speybroeck N, Xa NX, Thanh NN, Ky PV, et al. Longlasting insecticidal hammocks for controlling forest malaria: a communitybased trial in a rural area of central Vietnam. PLoS One. 2009;4:e7369.

38. Wilson AL, Hussey VC, Logan JG, Lindsay SW. Are topical insect repellents effective against malaria in endemic populations? A systematic review and meta-analysis. Malar J. 2014;13:446.

39. Kiszewski $A E$, Darling ST. Estimating a mosquito repellent's potential to reduce malaria in communities. J Vector Borne Dis. 2010;47:217-21.
40. Nsimba SED, Rimoy GH. Self-medication with chloroquine in a rural district of Tanzania: a therapeutic challenge for any future malaria treatment policy change in the country. J Clin Pharm Ther. 2005;30:515-9.

41. Kimani J, Phiri K, Kamiza S, Duparc S, Ayoub A, Rojo R, et al. Efficacy and safety of azithromycin-chloroquine versus Sulfadoxine-Pyrimethamine for intermittent preventive treatment of Plasmodium falciparum malaria infection in pregnant women in Africa: an open-label, randomized trial. PLoS One. 2016;11:e0157045.

42. Beeson JG, Boeuf $P$, Fowkes FJI. Maximizing antimalarial efficacy and the importance of dosing strategies. BMC Med. 2015;13:110.

43. Takahashi E, Nonaka D, Iwagami M, Vilay P, Chanthakoumane K, Kobayashi J et al. Patients' adherence to artemisinin-based combination therapy and healthcare workers' perception and practice in Savannakhet province, Lao PDR. Trop Med Health. 2018;64:44.

44. Pillai DR, Labbé AC, Vanisaveth V, Hongvanthong B, Pomphida S, Inkathone S, et al. Plasmodium falciparum malaria in Laos: chloroquine treatment outcome and predictive value of molecular markers. J Infect Dis. 2001;183:789-95.

45. Sserwanga A, Harris JC, Kigozi R, Menon M, Bukirwa H, Gasasira A, et al. Improved malaria case management through the implementation of a health facility-based sentinel site surveillance system in Uganda. PLoS One. 2011;6:e16316

46. Malaria Care. Improving malaria case management across the continuum of care in Ghana. 2014. https://malariacare.files.wordpress.com/2014/04/ malariacare-ghana-fact-sheet-2014.pdf. Accessed 1 July 2018.

47. Zurovac D, Githinji S, Memusi D, Kigen S, Machini B, Muturi A, et al. Major improvements in the quality of malaria case-management under the "test and treat" policy in Kenya. PLoS One. 2014;9:e92782.

48. President's Malaria Initiative: fighting malaria and saving lives. Providing a helping hand to remote villages in Burma: mobile malaria teams. 2013. https://www.pmi.gov/news/stories-from-the-field/stories-from-thefield\%2D\%2D-detail/providing-a-helping-hand-to-remote-villages-in-burmamobile-malaria-teams. Accessed 1 July 2018.

49. Malaria Consortium. Trans-border malaria programme. https://www. malariaconsortium.org/media-downloads/987/inFocus:\%20Transborder\%20malaria\%20programme\%20Cambodia. Accessed 2 July 2018.

50. Landier J, Parker DM, Thu AM, Lwin KM, Delmas G, Nosten FH, et al. Effect of generalised access to early diagnosis and treatment and targeted mass drug administration on Plasmodium falciparum malaria in eastern Myanmar: an observational study of a regional elimination programme. Lancet. 2018; 391:1916-26.

51. Adhikari B, Pell C, Phommasone K, Soundala X, Kommarasy P, Pongvongsa $T$, et al. Elements of effective community engagement: lessons from a targeted malaria elimination study in Lao PDR (Laos). Glob Health Action. 2017;10:1366136.

52. Sonkong $K$, Chaiklieng $S$, Neave $P$, Suggaravetsiri $P$. Factors affecting delay in seeking treatment among malaria patients along Thailand-Myanmar border in Tak Province, Thailand. Malar J. 2015;14:3.

53. Verschuere J, Decroo T, Lim D, Kindermans JM, Nguon C, Huy R, et al. Local constraints to access appropriate malaria treatment in the context of parasite resistance in Cambodia: a qualitative study. Malar J. 2017:16:81.

54. Neave PE, Soares ML. Barriers to malaria control in rural south-west TimorLeste: a qualitative analysis. WHO South East Asia J Public Health. 2014;3:41-5.

Ready to submit your research? Choose BMC and benefit from:

- fast, convenient online submission

- thorough peer review by experienced researchers in your field

- rapid publication on acceptance

- support for research data, including large and complex data types

- gold Open Access which fosters wider collaboration and increased citations

- maximum visibility for your research: over $100 \mathrm{M}$ website views per year

At $\mathrm{BMC}$, research is always in progress.

Learn more biomedcentral.com/submission 